\title{
Complete Analysis of the Impact of Distributed Heat Accumulators on the Efficiency of the District Heating Networks
}

\author{
Malgorzata Kwestarz, M.Sc., Ph.D. in Engineering \\ Warsaw University of Technology, Building Installation, Hydrotechnics and Environmental Engineering \\ Department, District Heating and Gas Systems Division 00-653 Warsaw, Nowowiejska Street 20, Poland
}

\begin{abstract}
The article concerns the analysis of the impact of distributed heat storages (accumulators) type TES (Thermal Energy Storage) on the parameters of the district heating network.

The simulation results show that the heat storage has a significant impact on the functioning quality of the heating system, reduces the time delay of transport and as well as minimizes heat loss during distribution process. The comparative analysis of simulation results and the data obtained from measurements confirmed the correctness of simulation studies.
\end{abstract}

Keywords: heat storage, heat accumulator, district heating network.

\section{Introduction}

In technical literature, sufficient studies concerning simulation of district heating networks are available. In [1], the study results reveal that the aim was to determine the most energy efficient DH water supply temperatures in the case networks. It was demonstrated that if the ring network design is utilized, the district heating system is easier to control. District Energy systems are reviewed and possible future enhancements involving thermal networks are considered in [2]. Also, the integration of combined heat and power with district energy permitting the cogeneration of electricity and heat is examined from several view points and for various locations and applications $[3,4,5]$. The modelling of the dynamic characteristics of a district heating network was investigated in [6,7,8]. Two important parameters and their mathematical expressions representing the dynamic characteristics of the $\mathrm{DH}$ network are analyzed. These parameters include the lag time and the relative attenuation degree of DH systems. The correctness of the dynamic models was verified based on comparison of the actual data with the calculated results of the two above mentioned parameters.

An approach for minimization of the capital costs and energy consumption in a district heating network was presented in [9] using a case study based on a district heating network in UK. A number of different design cases were simulated, taking into account different supply and return temperatures and target pressures losses. In [10], two methods of DH network dimensioning have been compared for the tree structure of the network. In the article, network dimensioning, heat losses and the basics of calculating the cost of electricity for pumping were presented.

Thermal energy storage (TES) allows excess thermal energy to be collected for lateral use, hours, days or months later, at individual building, multiuser building or even town district

depending on the specific technology. TES as an element of district heating network located near power station with CHP or supplied by renewable energy source can increase efficiency of the running of DH $[11,12,13,14,15]$. The influence of distributed heat storages TES type on parameters of operation of DH networks until now has not been analyzed. This article demonstrates the advantages of a distribution of heat storages in the area of a district heating network.

\section{Admission}

Heat storage popularly called accumulator was presented as part of the district heating network. It has been proven that in the era of market economy, where heat and electricity are a commodity, installation and operation of heat storages is necessary from a technical and economic point of view. The investment, which is the construction of heat storages is the answer to two questions: how big of heat capacity should be storage or storages group and what should be their location.

As far as the location of the accumulators is concerned, they are still built only in the immediate vicinity of the heat source.

In this article we proved that heat storages can be distributed in the area of district heating network. Each of them works in the maximum heat demand as a local source of heat and when the minimum partitions heat as an additional heat consumer. The size of the storage corresponds fully storage capacity. The selection of areas of cooperation heat storage with heat consumers is associated with the division of the district heating network subsystems, which will be supplied with heat at a constant average level during the day. Thus, the 
transmission network - the main will work with a constant load, which means a constant flow and minimal fluctuations in supply and return temperature supplying subsystems. The flow and temperature will change in a year, taking into account the summer, winter and two transitional periods.

It is assumed that the distributed heat storages increase the efficiency of the district heating network by reducing the time delay of the transport and heat losses during the distribution process.

The transport delay is the time period during which the heat is transported i.e. the hot water flows from the heat source to heat exchangers station (the final consumer).

The indicator delay of transport for the indicated heat exchangers station is the ratio of the delay transport with distributed heat storages to the time delay for network without distributed heat storages.

Heat loss of the heating system is called a difference in the stream of heat energy generated in the heat source and heat flux delivered to collection points $\dot{Q}^{\prime}{ }_{H P}$ :

$$
\dot{Q}_{\text {loss }}=\dot{Q}_{H P}-\dot{Q}_{H P}^{\prime}
$$

where:

$\dot{Q}_{\text {loss }}$ - Heat loss of the district heating system, W,

$\dot{Q}_{H P}$ - Flow of heat energy generated in the heat plant, W,

$\dot{Q}_{H P}^{\prime}$ - Flow of heat energy delivered to collection points, W.

An indicator of the loss of thermal power in the network is the ratio of the thermal power lost in the process of distribution heating network with distributed storage tanks to losses of thermal power in the network without trays.

\section{Simulation Of Mass Transport}

Computer simulation heating network with distributed storage tanks is used according to the Kirchhoff's $1^{\text {st }}$ and $2^{\text {nd }}$ law and the equation of flow.

In vector form Kirchoff's $1^{\text {st }}$ law is:

$$
\underline{A_{1}} \cdot \underline{\dot{M}}=\underline{L}^{*}
$$

where:

$\underline{A_{1}}=\left[a_{i j}\right]_{\left(n-n_{1}\right) * m}$ - incidence matrix loaded nodes and bows,

$\mathrm{n}$ - the number of nodes,

$\mathrm{m}$ - the number of bows,

$\mathrm{n} 1$ - number of distinguished nodes (source)

$\underline{\dot{M}}^{T}=\left[\dot{M}_{1}, \dot{M}_{2}, \ldots, \dot{M}_{m}\right]$ - vector mass flows of the heating medium in the network graph curves,

$\left(\underline{L}^{*}\right)^{T}=\left[\dot{L}_{1}^{*}, \dot{L}_{2}^{*}, \ldots, \dot{L}_{n-n_{1}}^{*}\right]$ - vector loads in the nodes of the network graph.

In vector form Kirchoff's $2^{\text {nd }}$ law is written as follows:

$$
\underline{B_{f}} \cdot \underline{\Delta p}=\underline{0}
$$

where:

$B_{f}=\left[b_{i j}\right]_{k * m}$ - incidence matrix mesh core and bows,

$\mathrm{k}$ - number mesh core,

$\underline{\Delta p^{T}}=\left[\Delta p_{1}, \Delta p_{2}, \ldots, \Delta p_{m}\right]-$ vector pressure drops in the bows.

In turn, the flow equation is of the form:

$$
\Delta p_{\text {loss }}=\frac{8}{d^{4} \pi^{2} \rho}\left(\lambda \frac{L}{d}+\sum \zeta\right) \dot{M}^{2}
$$

where: 
$\Delta p_{\text {loss }}$ - pressure drop, $\mathrm{Pa}$,

$\lambda$ - coefficient of linear resistance,

$\zeta$ - coefficient of local resistance,

$\mathrm{L}$ - the tube length, $\mathrm{m}$,

$\mathrm{D}$ - inner diameter of the pipe, $\mathrm{m}$,

$\rho$ - density, $\mathrm{kg} / \mathrm{m} 3$

$\dot{M}$ - mass flow, $\mathrm{kg} / \mathrm{s}$.

Loop method [16] using the following equations:

$$
\begin{aligned}
& \frac{A_{1}}{B_{f}} \cdot \underline{\dot{M}}=\underline{L}^{*} \\
& \frac{\Delta p}{\Delta p_{\text {loss }}}=\underline{0} \\
& =f(\dot{M})
\end{aligned}
$$

It is assumed that the starting point is exactly the Kirchhoff's $1^{\text {st }}$ law. The end result of the simulation values of the heating medium flow in the pipe, the pressure in the network nodes and the temperature at selected points in the network [16].

\section{The Distributed Thermal Energy Storage Type TES}

The distributed thermal energy storage is intended for short-term energy storage using water as a carrier. These cylinders receive hot water supply to the upper portion storage, while the same quantity of cold water from the bottom of the storage is discharged. To fully exploit the capacity of the cylinder, drainage of hot water from top of the storage and outflow of cold water from the bottom is maintained in the gravitational separation of hot and cold water so called thermal stratification with the thermocline. The process is called as the Discharging the battery (figure 1). The storage having an internal volume $\mathrm{V}$ can store heat $\mathrm{Q}_{\max }$ whose value can be calculated according to:

$$
\mathrm{Q}_{\max }=\mathrm{V}\left(\rho_{1} \mathrm{c}_{1} \mathrm{t}_{1}-\rho_{2} \mathrm{c}_{2} \mathrm{t}_{2}\right)
$$

where:

$\mathrm{V}$ - the internal volume of the storage,

$\mathrm{t}_{1}$ - water temperature at the top of the storage,

$t_{2}$ - temperature of the water in the lower part of the storage,

$c_{1}$ - specific heat of water at a temperature $t_{1}$,

c2 - specific heat of water at a temperature $t_{2}$,

$\rho_{1}$ - density of water at a temperature $t_{1}$,

$\rho_{2}$ - density of water at temperature $t_{2}$.

The figure 1 shows a schematic approach of distributed thermal energy storage connected to the district heating network [17]

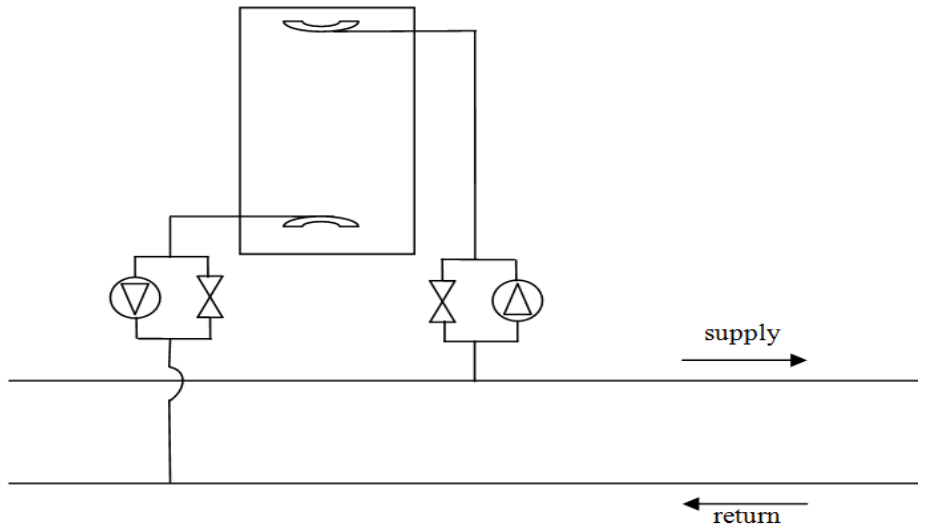

Fig. 1 Scheme of connection of distributed thermal energy storage and district heating network 


\section{Computer Simulation Of The Heating Network}

Simulation tests were carried out for existing - operated heating system. District heat supplied from a heat plant, provides heat for central heating and hot tap water to over 700 heat exchangers stations [17-23].

Simulations district heating network was made based on data obtained from measurement systems installed in the heat exchangers stations of district heating network. Verification of the model was based on a comparison of the results obtained by the simulation with real data measured at four points in the network:

- the input and output points of the heat source,

- at the heat exchangers stations of district heating network (consumption points):

A - working only for central heating $(\mathrm{CH})$,

$\mathrm{B}-\mathrm{CH}$ and tap water heating- hot tap water (HTW)

and $\mathrm{C}-\mathrm{CH}$ and HTW.

Consumptions points on the network graph shown on figure 2 .

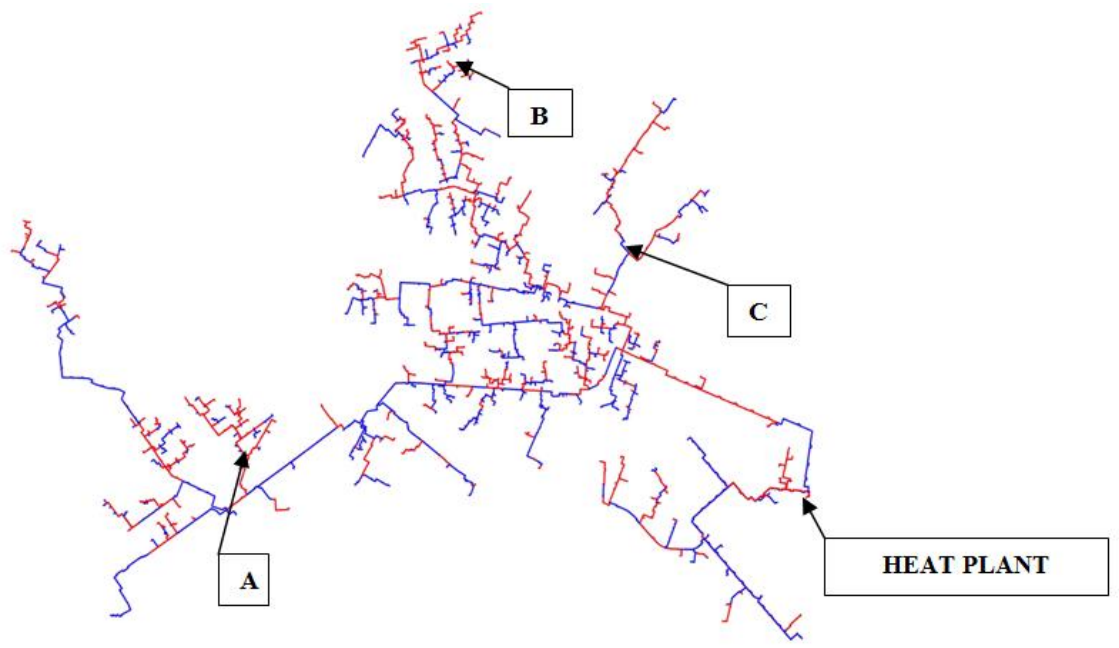

Fig. 2 Graph of district heating systems with measuring points

The input node heat source is compared with the return temperature and the mass flow of water flowing in the heat source and heat outputs. Analysis of the results showed the correctness of the model. Calculated relative errors are as follows:

1. The value of a water stream obtained by simulation vitiated 7, $3 \%$ for January $1,1 \%$ in March $0,4 \%$ in July and $0,1 \%$ for November.

2. The power source obtained by the simulation has an error at 3, 8\% for January 2, 8\% for March 2, 9\% for July and $8,0 \%$ for November.

3. The temperature of water returning to the source of the received simulation result deviates from the measured values on January 3, 6\%, 5 March, $1 \%$, July 4, November $0 \%$ and $6.5 \%$.

\section{Scenarios Simulation Of The Heating System}

Further analysis of the heat distribution network was carried out for the following variants:

1. Scenario WINTER - simulation for measurement data (parameters of district heating stations and sources)

Option 1 - the month of January,

Option 2 - the month of March,

Option 3 - the month of November.

2. Scenario SUMMER - working heating system solely for hot water.

Option 4 - simulation of the measurement data of July,

Option 5 - simulation for the $80 \%$ rate of capacity utilization exchangers hot tap water

Option 6 - simulation for the $20 \%$ rate of capacity utilization heat hot tap water.

In order to study the behavior of the heating network as characteristic points set - three district heating substations located furthest away from the heat source and the location of three points distributed heat storages. So far the places for heat storages have been selected in urban areas with concentrated multifamily houses.

Figure 3 shows a graph of the district heating network with marked characteristic points. 


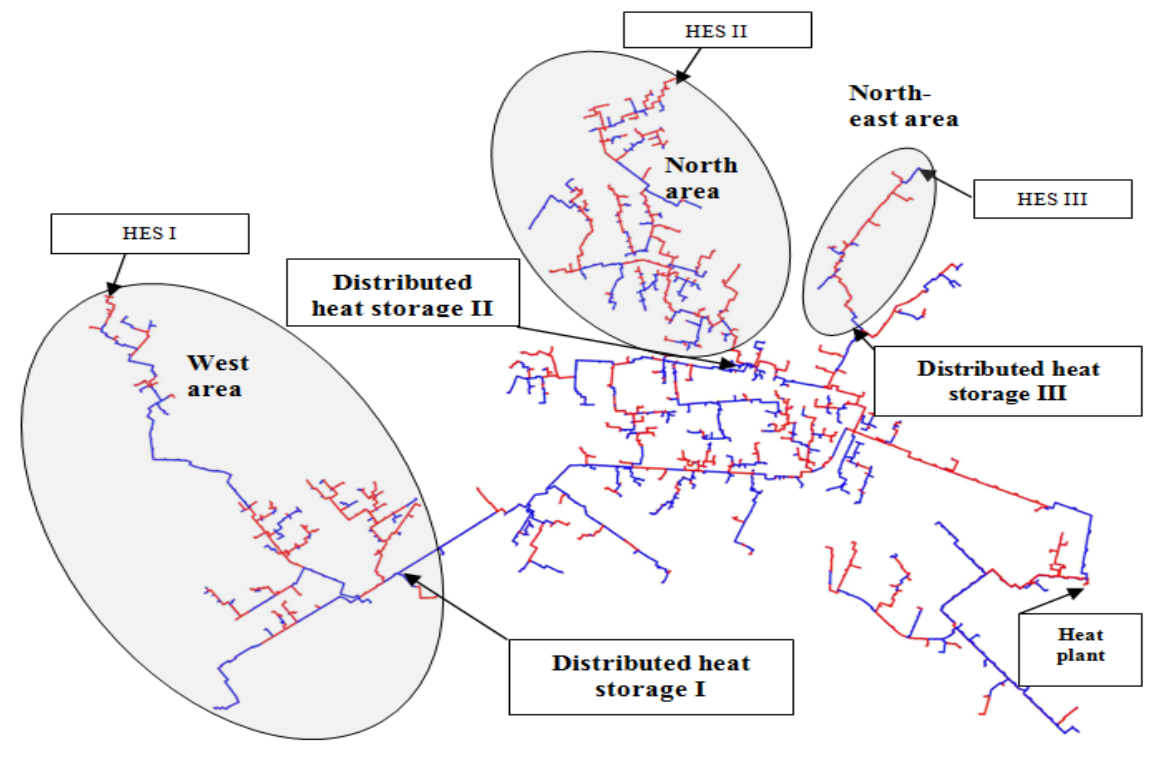

HES - heat exchangers' station/substation

Fig. 3 District heating network graph with marked characteristic objects

The delay transport for the analysed variants was calculated using simulation studies index. The results are shown in the graph below (Figure 4).

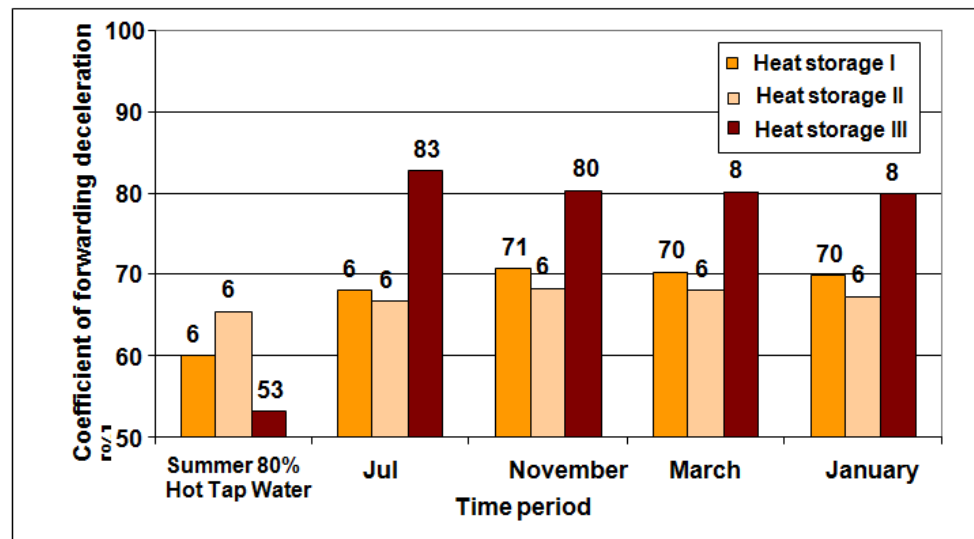

Fig. 4 Change the value of the indicator latency transport for the district heating network cooperating with distributed heat storages

When comparing the values for the ratio of transport delays, it can be seen clearly that cooperation heat storage I and II of the district heating system results in a permanent reduction of transport delays during the summer from $32 \%$ to $40 \%$ and in winter from $29 \%$ to $33 \%$. On contrary, the heat storage III in the summer, reduces the delay of transport by $47 \%$, and in winter from $17 \%$ to $20 \%$.

Comparison of changes in the value of the indicator indicates a transport delay shows the need to install distributed heat storages in district heating system and at the same time confirms their correct location in this particular case.

For each of the above-mentioned variants, the district heating network is compared to the power heat source and power heat losses in network. The results are shown on figure 5 for the winter months, and figure 6 for the summer period. 


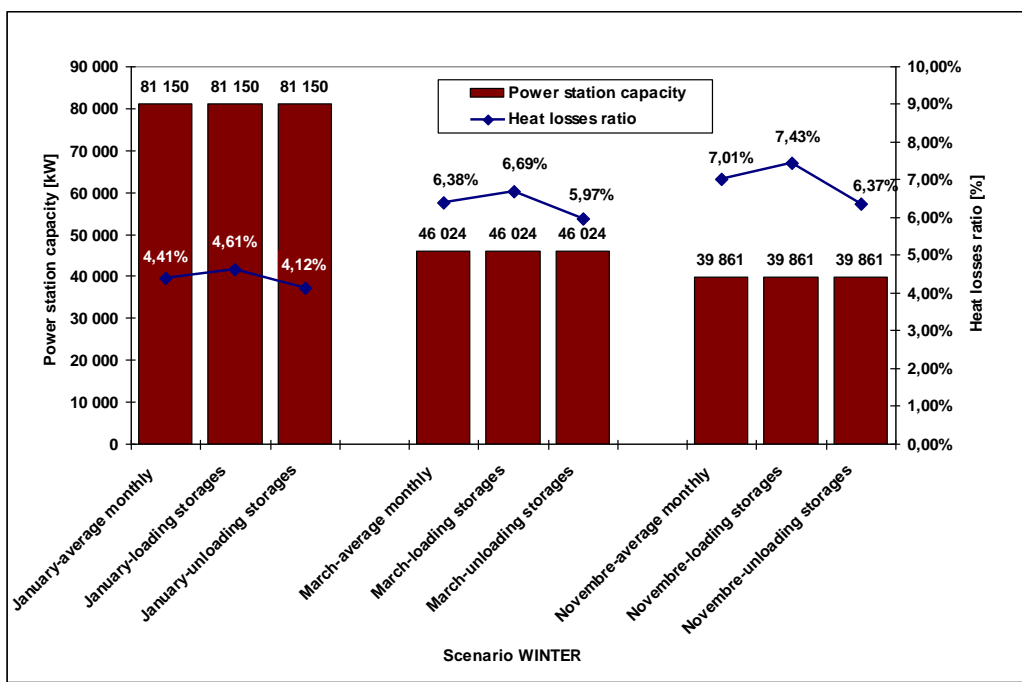

Fig. 5 Heat losses ratio in the process of distribution for the winter period

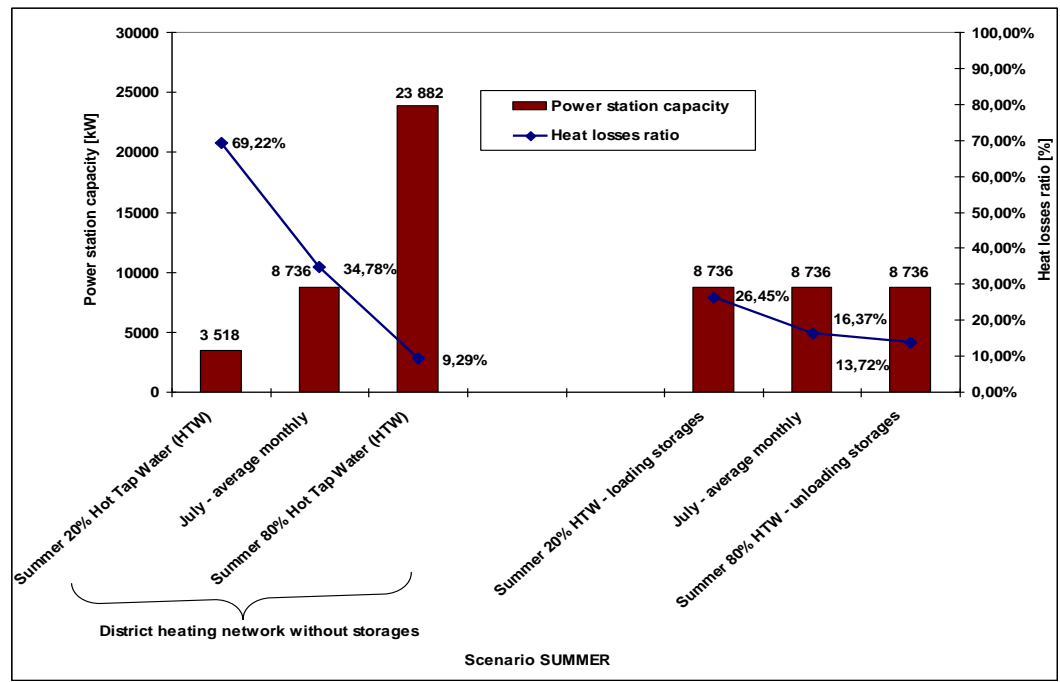

Fig. 6 Thermal capacity losses ratio in the distribution process for the summer period

Analysis of the results indicates that the distributed heat storage devices are essential in a modern district heating network especially during the summer when the heat is supplied dedicated only for hot tap water preparation. This period is characterized by high non-uniformity of heat consumption, which is an important factor in generating the heat losses throughout the district heating network. For the district heating network without distributed heat storages, the heat loss in a month of summer is at the level of $34.78 \%$. When the network is working with three distributed heat storages, the losses fall to $16.37 \%$. Of course, the losses are different for periods of network load acceptances and mode of heat storage (charging / discharging). The details are shown in the chart above (figure 6).

In the winter, i.e., during the heating season, reduction of the losses in the district heating network reaches about 5\%. This is due to the fact that the proportion of the heat loss is related to the whole sale and therefore the relative value is reduced over the summer period. It can be seen on figure 5 when the season of clothe source decreases.

The advantage of the use of distributed heat storages scattered in both summer and winter season is the ability to keep the heat load source at a constant level. This allows you to achieve maximum efficiency in the production of heat and thereby reduce pollutant emissions from combustion of fuel.

\section{Conclusion}

Analysis showed that the use of distributed heat storages, which are located at specific sites of the effect of reducing the delay time of the transport and reducing heat loss to the value, which is characterized by a heat network which does not have in its structure storages.

Installing distributed heat accumulators to better manage the heating network in the district heating system: 
- Dispersed heat storages in the district heating network increases the security of supply of consumers by creating subsystems with local heat sources which are the reservoirs of heat during the process of discharging;

- Cooperation with distributed heat storages stabilizes the load of the main district heating network, which translates into lower pumping costs, i.e. the work of circulation pumps and stabilizers,

- Working networks with permanent seasonal load allow you to control loads by qualitative method, which on one hand forces, but will allow for proper selection of temperature of the water supply and return water areas and this in turn results into a reduction of heat loss in the main network.

\section{Journal Pepers:}

\section{References}

[1]. Laajalehto T.,Kuosa M., Makila T., Lampinen M., Lahdelma K.: Energy efficiency improvements utilising mass flow control and a ring topology in a district heating network, Applied Thermal Engineering 69 (2-14), pp85-95

[2]. Rezaire B., Rosen M.A.: Sistrict heating and cooling: Review of technology and potential enhancements, Applied Energy 93 (1012), pp 2-10

[3]. Benonysson A., Bohm B., Ravn H.F.: Operational optimization in a district heating system, Energy Conservation and Management $36(5)$ pp.297-314 (1995)

[4]. Jing Z.X., Jiang X.S., Wu Q.H., Tang W.H., Hua B.; Modelling and optimal operation a small-scale integrated energy based district heating and cooling system, Energy 73 pp399-415 (2014)

[5]. Larsen H.V., Palsson H., Bohm B., Ravn H.F.: Aggregated dynamic simulation model of district heating networks, Energy Conservation and Management 43 (8) pp.995-1019 (2002)

[6]. Gabrielaitiene I., Bohm B., Sunden B. : Dynamic temperature simulation in district heating systems in Denmark regarding pronounced transient behavior, Journal Civil Engineering and Management, 17 (1), pp.79-87 (2011)

[7]. Jie P., Tian Z., Yuan S., Zhu N.: Modeling the dynamic characteristics of district heating network, Energy 39 (2012), pp126-134

[8]. Rosa, A., Boulter R., Church K., Svendsen, S. : District heating (DH) network design and operation toward a system-wide methodology for optimizing renewable energy solution (SMORES) in Canada: A case study Energy 45(1), pp. 960-974 (2012)

[9]. Pirouti M., Bagdanavicius A., Ekanayake J., Wu J., Jenkins N.: Energy consumption and economic analyses of district heating network, Energy 57 (2013) pp 149-159

[10]. Koiv T.-A., Mikola A., Palmistre.U: The new dimensioning method of the district heating network, Applied Thermal Engineering 71 (2014), pp78-82

[11]. Colella F., Sciacovelli A., Verda V.: Numerical analysis of medium scale latent energy storage unit for district heating systems, Energy 45 (1) pp. 397-406 (2012)

[12]. Tveit T.M.,Savola T., Gebremedhin A., Fogelholm C.:Multi-period MINLP model for optimizing operation and structural changes to CHP plants in district heating networks with long-term thermal storage, Energy Conservation and Management 50 (3) pp. 639647 (2009)

[13]. Vesoja E., Nikula H., Sierla S., Karhela T., Flikkema P.G., Yang C.W.: Hybrid modeling and co-simulation of district heating systems with distributed energy sources, Workshop on modeling and Simulation of Cyber-Physical Energy Systems, MSCPES 2014 - Held as Part of CPS week, Proceedings, art. No.6842395 (2014)

[14]. Vesterlund M., Dahl J.: A method for the simulation and optimization of district heating systems with meshed networks, Energy Conservation and Management 89, pp.555-567 (2015)

[15]. Zeghici R. M., Damian A., Frunzulica R., Iordache F.: Energy performance assessment of complex district heating systems which used gas-driven combined heat and power, heat pumps and high temperature aquifer thermal energy storage, Energy and Buildings 84, pp.142-151 (2014)

[16]. Kwestarz M: Heat storage in storages and their effect on the parameters of district heating network operation, District Heating, Heating, Ventilation, no. 11, (2012), ISSN: 0137-3676, pp 416-422

[17]. Osiadacz A.J., Kwestarz M..: Analysis of impact of a heat container on operating parameters of a district heating network, Energy Market no. 5 (102), (2012), ISSN 1425-5960, pp 59-66

[18]. Osiadacz A.J., Chaczykowski M., Kotyński Ł., The software package for simulating static heating networks, Heating, Ventilation,

Books: no.12/2012, ISSN: 0137-3676, pp 526-529

[19]. Osiadacz A.J: Simulation and analysis of gas network, London E\&F.N.SPON (1987)

Theses:

[20]. Kwestarz M: Analysis of impact of a heat storage on operating parameters of a district heating network, PhD. dissertation, Warsaw University of Technology, Warsaw (2011)

\section{Proceeding Papers:}

[21]. Chaczykowski M., Kwestarz M.: Computer simulation of a district heating network as an instrument to manage a contemporary heating company, 16th Technical Conference of the Polish District Heating Chamber of Commerce, Warsaw, November (2012)

[22]. Kwestarz M.: Heat storage in storages and their effect on the parameters of district heating network operation, 16th Forum of Polish Heating Engineers, Międzyzdroje, September (2012)

[23]. Osiadacz A.J., Kwestarz M: Computer simulation of a district heating network as an element in operator`s decision process, 3rd Technical Conference Zegrze (2010) 